\title{
P-0555-Association of reduced serum vitamin D levels with insulin resistance in Bangladeshi impaired glucose tolerance subjects
}

Afroz MS 1 , Hossain IA ${ }^{2}$, Azam MZ1, Khan MH', Ali L ${ }^{2}$

${ }^{1}$ Department of Biochemistry and Molecular Biology, Rajshahi University, Rajshahi, Bangladesh; ${ }^{2}$ Department of Biochemistry and Cell Biology, Bangladesh University of Health Sciences (BUHS), Dhaka, Bangladesh.

E-mail: slabonybio@yahoo.com

\section{Background}

-Impaired glucose tolerant (IGT) subjects, the subgroup of prediabetes in

Bangladeshi population have previously been shown to have insulin resistance; however, the underlying mechanism(s) of this defect is still unclear.

- Circulating vitamin D is known to play a role in insulin sensitivity by expressing the vitamin $\mathrm{D}$ binding protein in pancreatic cell.

- A number of studies have been conducted between the relationships of serum vitamin D levels with insulinemic indices in type 2 diabetic subjects but in IGT state, there is paucity of data regarding this association.

Aim

The present study has been explored to evaluate the levels of reduced vitamin D levels and its association with insulin resistance in IGT subjects.

Study Subjects

- Under a case-control design a total of 20 IGT subjects $(\mathrm{M} / \mathrm{F}, 9 / 11$; age in years, $46 \pm 9$; BMI in $\mathrm{kg} / \mathrm{m}^{2}, 25 \pm 3 ; \mathrm{M} \pm \mathrm{SD}$ ) and 27 age, sex and BMI- matched Healthy Controls (M/F, 14/13; age in years, $45 \pm 12$; BMI in $\mathrm{kg} / \mathrm{m}^{2}, 26.84 \pm 4.34$ ) were investigated. IGT was diagnosed as per WHO Study Group criteria following a 2 sample OGTT.

\section{Inclusion criteric}

-IGT subjects were purposively recruited from the BIHS General Hospital,

Darussalam, Dhaka, Bangladesh.

-None of the subjects had any history of using any antidiabetic agent or other medication including hormonal therapy known to affect on carbohydrate, lipid or insulin metabolism;

\section{Exclusion criteria}

Subjects suffering from any systemic illness like acute severe septic conditions, acute and chronic cardiac disease, hepatic, renal, acute and chronic respiratory failure, drugs affecting liver enzymes, cancer, stroke, type 1 diabetes, current medication and pregnant subjects was excluded from the study.

\section{Method:}

-Fasting and postprandial serum glucose were measured by glucose-oxidase method; -Lipid profile was measured by enzymaticcolorimetric method;

-Serum insulin and vitamin D were analyzed using commercially available kits by ELISA technique;

-Insulin resistance (HOMA-IR) was

calculated by homeostasis model assessment (HOMA);

\section{Acknowledgement}

-Bangladesh University of Health Sciences, Dhaka Bangladesh.

\section{References}

World Health Organization: Definition, diagnosi and classification of diabetes mellitus and it complications: Report of a WHO Consultation. Par 1. Diagnosis and classification of diabetes mellitus Geneva, World Health Organization, 1999.

\section{Results}

-HOMA-IR was significantly higher in IGT subjects compared to the control $(1.82 \pm 0.25$ vs. $1.02 \pm 0.65, p=0.006)$ (Table 1).

$\cdot[25(\mathrm{OH}) \mathrm{D}]$ was significantly lower in IGT subjects compared to the control $(18.23 \pm 2.25 \mathrm{vs} .26 .35 \pm 4.65 \mathrm{ng} / \mathrm{ml}$, $\mathrm{p}=0.013$ ) (Table 1).

-In regression curve analysis, [25(OH)D] showed significant correlation with fasting serum insulin $\left(\mathrm{r}^{2}=0.435\right.$, $\mathrm{p}=0.004)$ and HOMA-IR $\left(\mathrm{r}^{2}=0.396, \mathrm{p}=0.007\right)$ in IGT subjects (Figure 1).

-In multiple linear regression analysis, HOMA-IR showed significant negative association with $[25(\mathrm{OH}) \mathrm{D}](\beta=$ $0.261, \mathrm{p}=0.038$ ) in IGT subjects after adjusting the effects of major confounding variables of body mass index (BMI), triglyceride (TG) and $\mathrm{HbA}_{1 \mathrm{c}}$ respectively (Table 2).

Table 1: Anthropometric, clinical and biochemical characteristics of the study subjects

\begin{tabular}{lccc}
\hline Variables & $\begin{array}{c}\text { Control } \\
\text { Subjects }(\mathrm{n}=27)\end{array}$ & $\begin{array}{c}\text { IGT } \\
\text { Subjects }(\mathrm{n}=20)\end{array}$ & P Value \\
\hline Age (years) & $45 \pm 12$ & $46 \pm 9$ & 0.052 \\
Body mass index $\left(\mathrm{kg} / \mathrm{m}^{2}\right)$ & $27 \pm 11$ & $25 \pm 3$ & 0.593 \\
Waist to hip ratio & $0.97 \pm 0.1$ & $0.95 \pm 0.0$ & 0.426 \\
Percent body fat & $27 \pm 15$ & $30 \pm 6$ & 0.308 \\
Systolic blood pressure $(\mathrm{mmHg})$ & $118 \pm 9$ & $115 \pm 12$ & 0.224 \\
Diastolic blood pressure $(\mathrm{mmHg})$ & $76 \pm 6$ & $75 \pm 10$ & 0.746 \\
Fasting serum glucose $(\mathrm{mmol} / \mathrm{l})$ & $5.0 \pm 0.5$ & $5.0 \pm 0.4$ & 0.992 \\
Postprandial serum glucose $(\mathrm{mmol} / \mathrm{l})$ & $6.3 \pm 0.8$ & $9.2 \pm 0.9$ & $<0.001$ \\
HbA ${ }_{1 \mathrm{C}}(\%)$ & $5.8 \pm 0.5$ & $5.7 \pm 0.5$ & 0.713 \\
Total cholesterol (mg/dl) & $188 \pm 44$ & $201 \pm 49$ & 0.308 \\
Triglyceride (mg/dl) & $133 \pm 46$ & $181 \pm 65$ & 0.002 \\
HDL-cholesterol (mg/dl) & $39 \pm 14$ & $33 \pm 11$ & 0.103 \\
LDL- cholesterol (mg/dl) & $119 \pm 39$ & $121 \pm 47$ & 0.839 \\
Serum creatinine $(\mathrm{mg} / \mathrm{dl})$ & $0.94 \pm 0.1$ & $0.96 \pm 0.1$ & 0.001 \\
Serum alanine transaminase $(\mathrm{IU} / \mathrm{L})$ & $26 \pm 9$ & $37 \pm 11$ & 0.001 \\
Fasting serum insulin $(\mu \mathrm{IU} / \mathrm{ml})$ & $13.5 \pm 1.32$ & $8.65 \pm 3.21$ & $<0.001$ \\
HOMA-IR & $1.02 \pm 0.65$ & $1.82 \pm 0.25$ & 0.006 \\
[25(OH)D] (ng/ml) & $26.35 \pm 4.65$ & $18.23 \pm 2.25$ & 0.013 \\
\hline
\end{tabular}

Results were expressed as mean $( \pm \mathrm{SD})$. $\mathrm{n}=$ number of subjects; IGT, impaired glucose tolerance; HOMA-IR, insulin resistance assessed by homeostasis model assessment; [25(OH)D], 25-hydroxyvitamin D.

Figure 1: Regression curve analysis to see the relationship of serum leptin with insulinemic indices among the study subjects

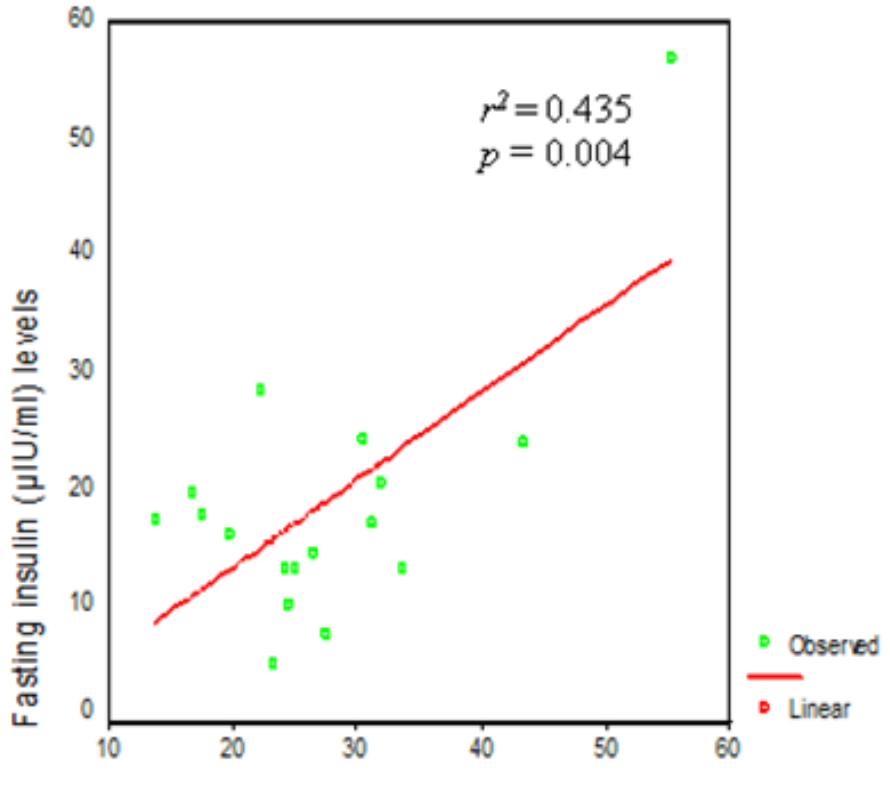

Serum vitamin D $(n g / m)$ lev els

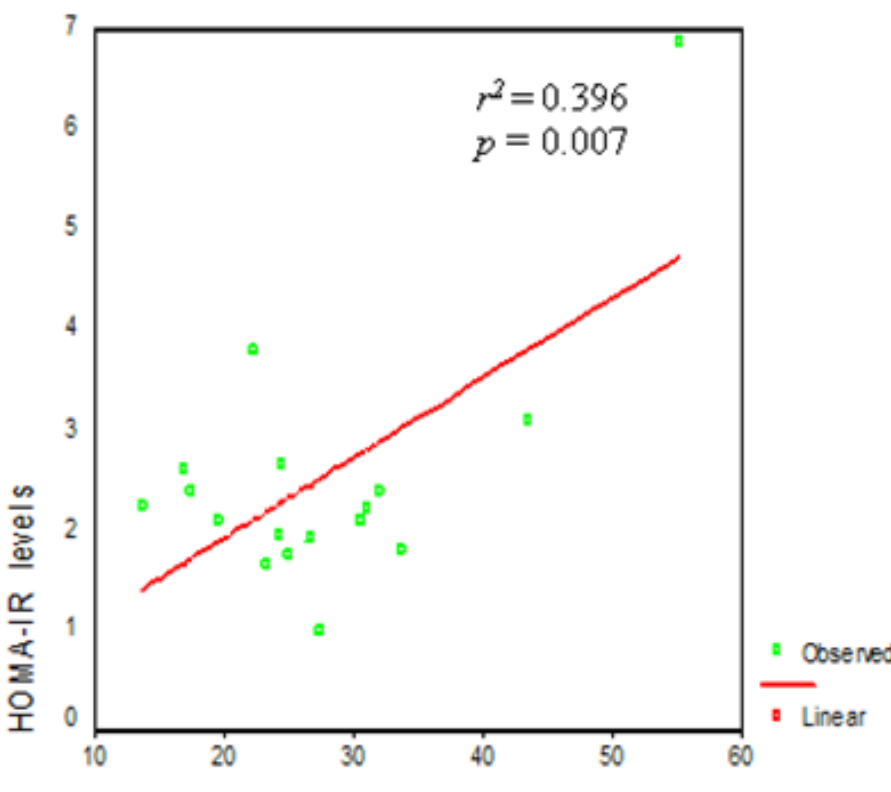

Serum vitamin D $(\mathrm{ng} / \mathrm{ml})$ lev els
Table 2: Multiple linear regression analysis to evaluate the association of HOMA-IR with [25(OH)D] levels in IGT subjects after adjusting the effects of major confounders.

\begin{tabular}{lccc}
\hline \multirow{2}{*}{ Variables } & \multicolumn{3}{c}{ Dependent variable: HOMA-IR } \\
\cline { 2 - 4 } & Regression coefficient $\boldsymbol{\beta}$ & t value & P value \\
\hline Constant & $*$ & 0.852 & 0.399 \\
Body mass index $\left(\mathrm{kg} / \mathrm{m}^{2}\right)$ & 0.291 & 1.093 & 0.280 \\
Percent body fat & 0.021 & -0.076 & 0.940 \\
Triglyceride $(\mathrm{mg} / \mathrm{dl})$ & 0.148 & -1.104 & 0.275 \\
$\mathrm{HbA}_{1 \mathrm{C}}(\%)$ & 0.042 & -0.336 & 0.738 \\
{$[25(\mathrm{OH}) \mathrm{D}](\mathrm{ng} / \mathrm{ml})$} & -0.261 & 2.131 & 0.038 \\
\hline
\end{tabular}

Adjusted $\mathrm{R}^{2}=0.310$; the level of significance at $\mathrm{p}<0.05 ; \beta$, regression coefficient; CI, confidence interval; $\mathrm{HbA}_{1 \mathrm{c}}$, glycated hemoglobin; IGT, impaired glucose tolerance; HOMA-IR, insulin resistance assessed by homeostasis model assessment; [25(OH)D], 25-hydroxyvitamin D.

\section{Conclusion}

From the above study results, it may conclude that circulating vitamin D levels is reduced in IGT subjects and this reduction is seemed to be mediated by insulin resistance among these subjects. 